\title{
Chondrogenic Differentiation of Human Mesenchymal Stem Cells in Three-Dimensional Alginate Gels
}

\author{
JINPING XU, M.D., ${ }^{1}$ WEI WANG, Ph.D., ${ }^{1}$ MATT LUDEMAN, Ph.D., ${ }^{2}$ KEVIN CHENG, Ph.D., ${ }^{2}$ \\ TAKAYUKI HAYAMI, Ph.D., ${ }^{1}$ JEFFREY C. LOTZ, Ph.D., ${ }^{2}$ and SUNIL KAPILA, D.D.S., Ph.D. ${ }^{1}$
}

\begin{abstract}
We characterized the temporal changes in chondrogenic genes and developed a staging scheme for in vitro chondrogenic differentiation of human mesenchymal stem cells (hMSCs) in three-dimensional (3D) alginate gels. A time-dependent accumulation of glycosaminoglycans, aggrecan, and type II collagen was observed in chondrogenic but not in basal constructs over 24 days. qRT-PCR demonstrated a largely characteristic temporal pattern of chondrogenic markers and provided a basis for staging the cellular phenotype into four stages. Stage I (days 0-6) was defined by collagen types I and VI, Sox 4, and BMP-2 showing peak expression levels. In stage II (days 6-12), gene expression for cartilage oligomeric matrix protein, HAPLN1, collagen type XI, and Sox 9 reached peak levels, while gene expression of matrilin 3, Ihh, Homeobox 7, chondroadherin, and WNT 11 peaked at stage III (days 12-18). Finally, cells in stage IV (days 18-24) attained peak levels of aggrecan; collagen IX, II, and X; osteocalcin; fibromodulin; PTHrP; and alkaline phosphatase. Gene profiles at stages III and IV were analogous to those in juvenile articular and adult nucleus pulposus chondrocytes. Gene ontology analyses also demonstrated a specific expression pattern of several putative novel marker genes. These data provide comprehensive insights on chondrogenesis of hMSCs in 3D gels. The derivation of this staging scheme may aid in defining maximally responsive time points for mechanobiological modulation of constructs to produce optimally engineered tissues.
\end{abstract}

\section{INTRODUCTION}

A RTICULAR CARTILAGE is an avascular connective tissue populated by highly specialized cells of mesenchymal origin. These chondrocytes are responsible for producing, sustaining, and degrading an extensive cartilage-specific extracellular matrix (ECM). ${ }^{1}$ The ECM in healthy cartilage provides it with the shape stability and strength to sustain the complex loads that facilitate smooth skeletal movement. In spite of its durability, mature cartilage is vulnerable to injuries and disease processes that cause irreversible tissue damage because of its limited capacity for self-repair. ${ }^{2}$ Attempts to augment cartilaginous healing responses in both experimental animal models and arthritis patients have uti- lized two fundamentally different strategies, namely, (1) stimulation of regeneration in situ (e.g., periosteal and perichondral tissue grafting, subchondral penetration, artificial matrices, chondrogenic cell transplantation, and growth factor utilization) or (2) transplantation of replacement tissues (e.g., autografts and allografts). ${ }^{2-8}$ These methods generally produce fibrous tissue that lacks the desired mechanical and morphological characteristics of normal articular cartilage.

Recently, cell-based techniques that utilize a threedimensional (3D) carrier mimicking the physiological milieu are demonstrating promise. These approaches incorporate cells such as chondrocytes, periosteocytes, or perichondrocytes into a variety of carrier systems including alginate,

\footnotetext{
${ }^{1}$ Department of Orthodontics and Pediatric Dentistry, The University of Michigan School of Dentistry, Ann Arbor, Michigan.

${ }^{2}$ Department of Orthopaedic Surgery, University of California, San Francisco, California.
} 
fibrin, hyaluronate, collagen gels, or biodegradable macromolecular polymers. ${ }^{9-16}$ Additionally, numerous growth factors and cytokines, such as members of transforming growth factor superfamily (TGF- $\beta_{1},-\beta_{2}$, and $-\beta_{3}$; BMP-2, -6 , and -7), have demonstrated an encouraging capacity for chondrogenic induction. ${ }^{17-20}$ Unfortunately, the slow in vitro propagation of adult chondrocytes has limited their widespread use in cartilage regeneration techniques.

Human mesenchymal stem cells (hMSCs) have become a popular choice for cartilage tissue engineering given their capacity of rapid in vitro proliferation and lineage differentiation into chondrocytes. ${ }^{17-19}$ Because hMSCs encapsulated in alginate beads can form cartilage, this system is used commonly to study the molecular mechanisms involved in chondrogenesis and endochondral ossification. ${ }^{18,19}$ While the progression of chondrogenic differentiation has been generally described for this system, cellular phenotypes and temporal patterns of relevant gene expression have not been presented quantitatively or comprehensively. Such data would provide a solid foundation upon which to rationally explore and optimize in vitro factors that guide chondrogenic differentiation, such as mechanical or biologic manipulation.

To better understand the process of chondrogenic differentiation of hMSCs in a 3D carrier system amenable to in vitro manipulation, and to better define temporal aspects of relevant gene expression and phenotypic changes, we studied differentiating hMSCs in alginate gel discs at four distinct time points ranging from day 0 to 24 , using both traditionally defined markers and global gene expression profiles. Our results present a well-characterized system in which hMSCs in 3D alginate culture system demonstrate distinct stages of chondrogenic differentiation.

\section{MATERIALS AND METHODS}

\section{hMSC-alginate gel/cell cultures}

hMSCs, cell culture media, and supplements were obtained from Cambrex Bio Science (Walkersville, MD), and chemical reagents were purchased from Sigma-Aldrich (St. Louis, MO) unless otherwise mentioned. On the basis of their surface markers and their capability for multipotential differentiation, ${ }^{21}$ these cells have been previously characterized as stem cells. The capacity of the cells used in our experiments to undergo multipotential differentiation (chondrogenesis, osteogenesis, and adipogenesis) under appropriate conditions was further confirmed by preliminary studies (data not shown).

hMSCs were expanded in monolayer culture by passaging three times in MSC basal medium (MSCBM) with 10\% FBS. The cells $\left(10^{6}\right.$ cell $\left./ \mathrm{mL}\right)$ were suspended in $2 \%$ alginate $(\mathrm{w} / \mathrm{v})$ solution, the suspension was loaded into dialysis tubing (10,000 molecular weight cut-off) (Spectrum Laboratories, Rancho Dominguez, CA), and the tubing was soaked in $10^{2} \mathrm{mM}$ calcium chloride with swirling for $40 \mathrm{~min}$. The gelled constructs were washed with HEPES solution, and cut into cylindrical discs ( $6 \mathrm{~mm}$ in diameter and $2 \mathrm{~mm}$ in height). After recovery in MSCBM for $24 \mathrm{~h}$, considered day 0 , the cell/gel constructs were separated into two groups: the chondrogenic constructs were cultured in serum-free hMSC chondrogenic medium containing $10^{-7} \mathrm{M}$ dexamethasone, $50 \mu \mathrm{g} / \mathrm{mL}$ ascorbate, $50 \mathrm{mg} / \mathrm{mL}$ Insulin-transferrin sodium selenite, $100 \mu \mathrm{g} / \mathrm{mL}$ sodium pyruvate, $40 \mu \mathrm{g} / \mathrm{mL}$ proline, $2 \mathrm{mM}$ L-glutamine, and $10 \mathrm{ng} / \mathrm{mL}$ TGF- $\beta_{3}$; the baseline controls were cultured in MSCBM plus $10 \%$ FBS. The medium was changed every other day, and constructs were retrieved at days $0,6,12,18$, and 24 of culture for assaying.

\section{Cell proliferation and viability}

The proliferation of hMSCs was assayed using alamarBlue assay kit (Biosource, Camarillo, CA). Briefly, cell/gel constructs were washed in PBS and incubated with $300 \mu \mathrm{L}$ alamarBlue solution for $1 \mathrm{~h}$. The fluorescence of reduced alamarBlue in the solution was determined using a spectrophotometer (Molecular Devices, Sunnyvale, CA) at wavelengths of 530-nm excitation and 600-nm emission. After PBS wash, hMSCs were released by incubating gels with $55 \mathrm{mM}$ sodium citrate solution and the cell lysates were subjected to BCA protein assay (Pierce, Rockford, IL) to standardize alamarBlue fluorescence data.

Cell viability was assayed by LIVE/DEAD staining as per the manufacturer's instructions (Molecular Probes, Sunnyvale, CA). The central parts of constructs were sliced (0.1mm thick), washed with HBSS, and staining solution added. After 20-min incubation in darkness, the slices were washed with HBSS before observation under a fluorescent microscope (Nikon TS100, Tokyo, Japan). Cell viability was semiquantified as the percentage of live (green-stained) cells relative to the total number of live and dead (red-stained) cells in four randomly selected fields in each of four slices from each group.

\section{Histological assessment of chondrogenic differentiation}

The distribution of hMSCs in constructs and the cellular morphology were examined using hematoxylin and eosin (H\&E) staining. Newly synthesized cartilage ECM glycosaminoglycans (GAGs) were visualized by Safranin-O staining. Briefly, paraformaldehyde-fixed constructs were encased in agarose and then embedded in paraffin, sectioned and subjected to routine Safranin-O staining as described previously, ${ }^{9,19}$ and mounted with coverslips prior to photomicrography.

To localize collagen type II and aggrecan, sections were rehydrated prior to immunostaining with Histostain-SP kit (Invitrogen, Carlsbad, CA). The epitopes for aggrecan and type II collagen were exposed by treating the sections with either chondroitinase $\mathrm{ABC}$ or pepsin, respectively. Endogenous peroxidase was then quenched, and the sections were 
incubated with a blocking solution, followed by incubation with primary antibodies specific for human collagen type II (Chemicon International, Temecula, CA) or aggrecan (Abcam, Boston, MA). Sections were washed, incubated with biotinylated secondary antibody, washed again, and incubated with a streptavidin-peroxidase conjugate. Following further washing, sections were incubated with diaminobenzidine solution, and counterstained with hematoxylin. Negative control sections were incubated with nonimmune serum.

\section{Quantitative real-time PCR ( $q R T$-PCR) and RT-PCR analyses}

Total RNA was extracted from constructs, and from freshly liberated primary human juvenile articular chondrocytes, fibroblast-like annulus fibrosus cells, and chondrocyte-like nucleus pulposus cells of adult human intervertebral discs ${ }^{22}$ using RNeasy Mini kit (Qiagen, Valencia, CA). The latter three cell types were used to provide comparative data for the differentiating hMSCs. Juvenile chondrocytes (kindly provided by ISTO Technologies, St. Louis, MO) were harvested from articular cartilage of human donors ranging in age from birth to 3 years as previously described. ${ }^{23}$ Adult nucleus pulposus and annulus fibrosus cells were isolated from normal tissues collected at the time of spine fusion surgery (29-yearold male [nucleus] and 51-year-old male [annulus]) after approval by the UCSF institutional review board.

Because of the small size of the gels and low RNA yield, RNA was pooled from each of 20 chondrogenic or 20 basal constructs. Total RNA $(1 \mu \mathrm{g})$ from the constructs and from primary cells was reverse transcribed using Ominiscript RT Kit (Qiagen). A 1:100 dilution of the resulting cDNA was utilized as a template to quantify the relative content of mRNA by qRT-PCR (ABI PRISM 7500, Applied Biosystems, Carlsbad, CA) for the genes listed in Table 1. The specific primers designed with Primer Express 2.0 (Applied Biosystems) are also listed in this table. PCR reactions were performed with SYBR green PCR master mix kit (Applied Biosystems). $18 \mathrm{~S}$ rRNA was used as an internal control. Cycle threshold values of the genes of interest and the quantitative gene expression levels relative to $18 \mathrm{~S}$ rRNA in the samples were determined as per ABI System instructions.

RT-PCR was employed to validate the expression pattern of key cartilage-marker genes, including collagen types II $\alpha 1$ and $\mathrm{X} \alpha 1$, cartilage oligomeric matrix protein (COMP), and aggrecan in both basal and chondrogenic hMSCs. The procedures for reverse transcription of total RNA and subsequent dilution of synthesized cDNA were performed as described above. PCR amplification was performed using Taq PCR master mix kit (Qiagen) using the following conditions: $94^{\circ} \mathrm{C}$ for $4 \mathrm{~min}$, followed by 25 cycles at $94^{\circ} \mathrm{C}$ for $25 \mathrm{~s}, 53^{\circ} \mathrm{C}$ for $30 \mathrm{~s}$, and $72^{\circ} \mathrm{C}$ for $45 \mathrm{~s}$, and final extension at $72^{\circ} \mathrm{C}$ for $10 \mathrm{~min}$. Human glyceraldehyde-3-phosphate dehydrogenase (GAPDH) was used as an internal control. The specific primers used for RT-PCR were as follows: collagen type II $\alpha 1,5^{\prime}$-ACC CCA ATC CAG CAA ACG TT-3' (forward) and 5'-ATC TGG ACG TTG GCA GTG TTG-3' (reverse); collagen type $\mathrm{X} \alpha 1,5^{\prime}$-AGG TGC CAA AGG GGA ACA AG-3' (forward) and 5'-AAT CCT GGA ATG CCT GGT GG-3' (reverse); COMP, 5'-AGG ACA ACT GCG TGA CTG TG-3' (forward) and 5'-GTG TCC TTT TGG TCG TCG TT- $3^{\prime}$ (reverse); aggrecan, $5^{\prime}$-CCT CTG GAC AAC CAG GTG TT-3' (forward) and $5^{\prime}$-AAA CCA GGT CAG GGA CTC CT-3' (reverse); GAPDH, 5'-GAG TCA ACG GAT TTG GTC GT-3' (forward) and $5^{\prime}$-TTG ATT TTG GAG GGA TCT CG-3' (reverse).

\section{Microarray analysis}

As a complement to the qRT-PCR experiments, and to identify putative novel marker genes that may be specific to particular hMSC chondrocytic differentiation stages, we analyzed global gene expression patterns by microarray (Human U133 plus 2.0. containing 54,675 probe sets plus 62 control sets; Affymetrix, Santa Clara, CA). Total RNA was pooled from 20 constructs and cleaned by using RNeasy Mini kit (Qiagen) and assayed to confirm quality (Agilent 2100 BioAnalyzer, Santa Clara, CA). Double-stranded cDNA was prepared using an oligo(dT)-T7 RNA polymerase primer. Biotinylated cRNA was prepared by in vitro transcription, and the resulting RNA was fragmented by partial hydrolysis. Hybridization signal was obtained by staining with phycoerythrin-streptavidin (GeneChip Fluidics Station, Affymetrix). The tagged cRNA was hybridized to microarrays, and differential gene expression levels were assessed using Affy/affy PLM package and RMA (robust multiarray average) intensity in $\log 2$ scale for each probe set (gene). Essentially, the 11 perfect-match intensities per probe set were (1) background corrected, (2) quantile normalized, and (3) summarized for each probe set using a robust fit of linear models as described. ${ }^{24} \mathrm{~A}$ total of nine arrays were used, one for each condition and time point. Genes with contradictory multiple positive probes sets were not included in the results. Ontological analysis was performed using GOstat and DAVID software and the Gene-Ontology database publicly available through the Gene Ontology Project. ${ }^{25}$

To identify genes that may be putative unique markers for each differentiation stage, we first identified genes that were more than twofold up- or downregulated in chondrogenic media relative to basal media at the same time point. We further identified genes that were at least twofold more upor downregulated at one stage relative to any other stage. Because many genes fit these criteria for days 6 and 24, while few did for days 12 and 18, we refined the selection criterion to exclude genes less than fivefold up- or downregulated at days 6 and 24. This resulted in a manageable number of genes for all time points. These data were also utilized to derive gene ontology patterns.

\section{Statistical analysis}

Proliferation of hMSCs at individual time points in both basal and chondrogenic group was compared to that on day 0 


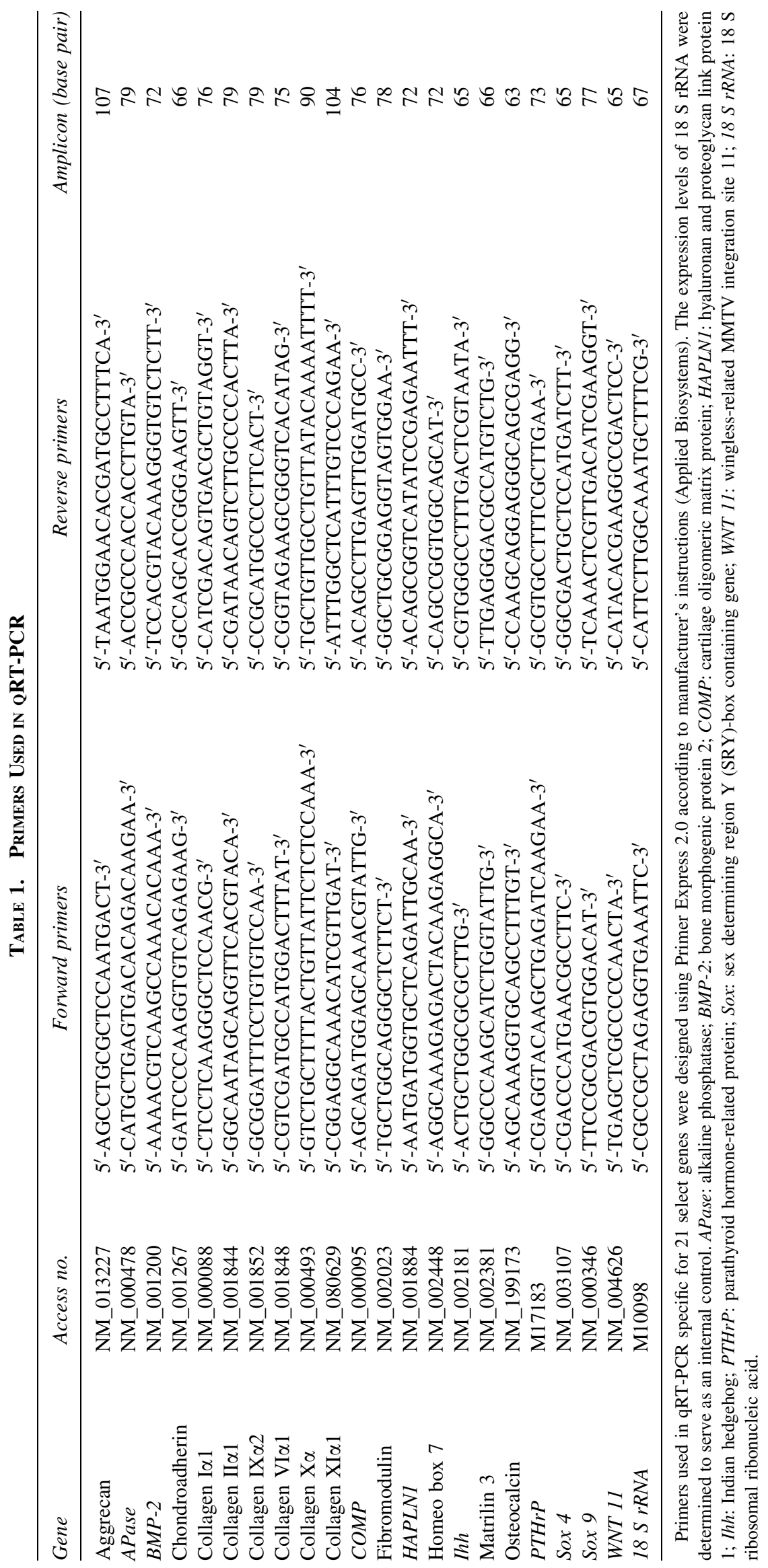



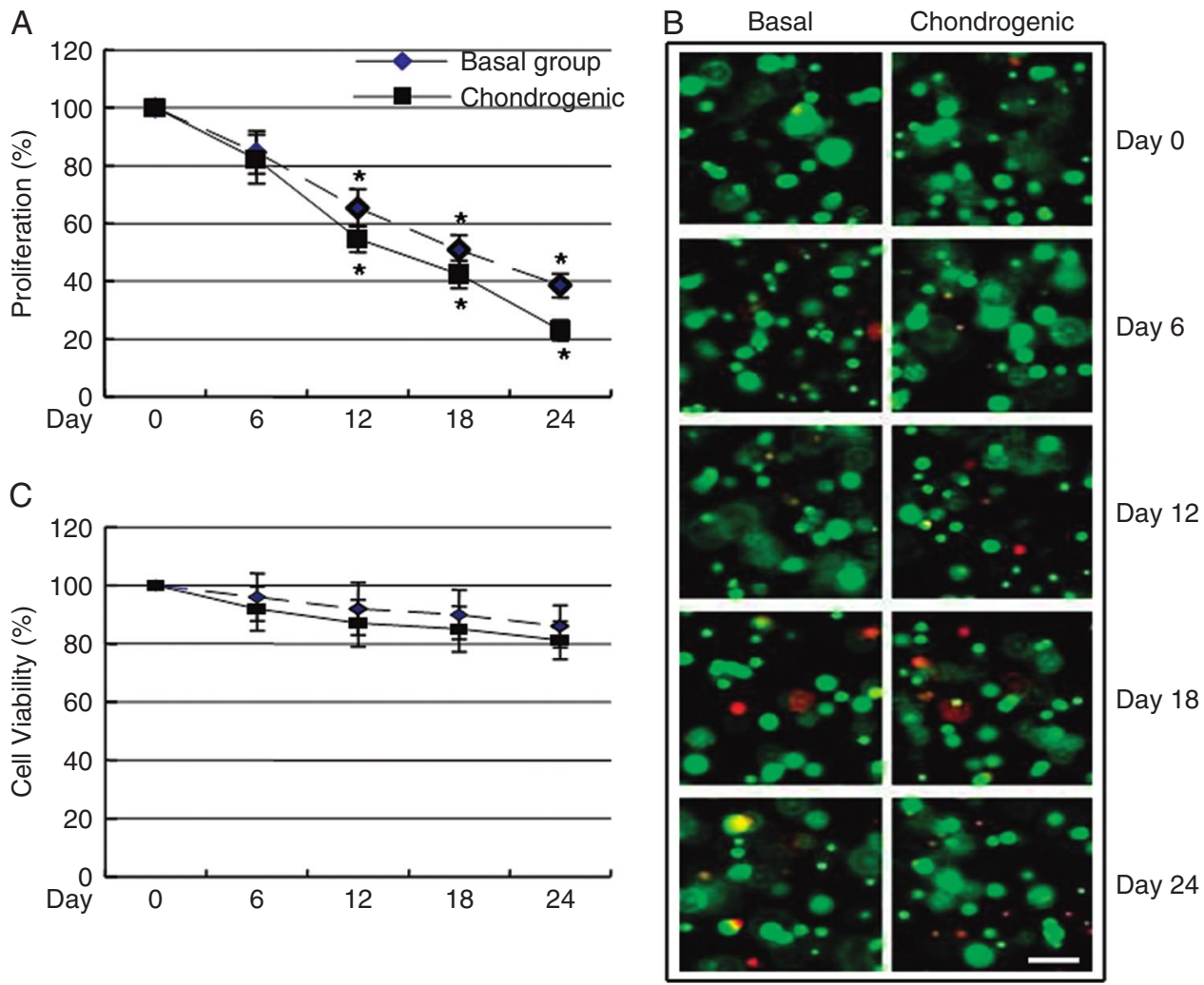

FIG. 1. hMSCs in alginate gels show decreased proliferation and minimal changes in cell viability over 24 days of culture. The proliferation of hMSCs in alginate gels, determined by alamarBlue staining, was significantly reduced from day 0 to 24 in both basal and chondrogenic constructs (A). However, there was no significant difference in cell proliferation between the two groups at any of the time points. (B) LIVE/DEAD fluorescent staining performed on slices of constructs showed a few dead cells (red) as compared to live cells (green). Semiquantitative analysis of these sections showed relatively stable cell viability in both groups from day 0 to 24 (C). Data were obtained from at least four cell/gel constructs, and expressed as mean $\pm \mathrm{SD}(* p \leq 0.01 \mathrm{vs}$. proliferation on day 0 ; magnification $\times 10$; bar represents $100 \mu \mathrm{m}$ ).

using single factorial analysis of variance (ANOVA). Gene expression of aggrecan, COMP, collagen type II, and collagen type $\mathrm{X}$ on days $6,12,18$, and 24 , respectively, in chondrogenic group and their corresponding time point in basal group were analyzed using Student's $t$ test.

\section{RESULTS}

\section{hMSCs show mildly diminished viability and progressive reduction in proliferation in long-term $3 D$ cultures}

Cell proliferation was slightly, but not significantly, lower at day 6 in both the basal and chondrogenic media relative to the baseline controls (Fig. 1A). In contrast, constructs cultured in both media had significantly lower cell proliferation at days 12,18 , and 24 relative to that at day 0 . By day 24 , the proliferation was reduced to $61.5 \%$ for the basal group and $76.9 \%$ for the chondrogenic group relative to day 0 cultures.

Under both basal and chondrogenic conditions, while a small number of dead cells were observed, cell viability remained relatively stable during the experimental period (Fig. 1B). Relative to baseline levels on day 0, there was a gradual but insignificant reduction in cell viability of $14 \%$ in basal group and $18 \%$ in chondrogenic group on day 24 (Fig. $1 \mathrm{C})$. The cell viability and proliferation showed no statistically significant differences between the basal and chondrogenic media at any time point.

\section{Cartilage-specific ECMs are accumulated in TGF- $\beta_{3}$-stimulated hMSC/gel constructs}

We next utilized histology and immunohistochemistry to assess the temporal changes in cell morphology and distribution of cartilaginous markers in constructs. H\&E staining showed homogenous cell distribution in both basal and chondrogenic constructs (Fig. 2A). While there was little change in cellular morphology in basal conditions, cells in chondrogenic media were observed to have large nuclei and became progressively bigger and more rounded over 24 days of culture. Clear pericellular lacunae were also observed in the chondrogenic construct but not in the basal construct. Consistent with these findings, Safranin-O staining demonstrated 
a temporal increase of staining in chondrogenic constructs, indicating increasing accumulation of GAGs (Fig. 2B). In contrast to the chondrogenic constructs, none of the basal constructs showed positive staining with Safranin-O.

Immunohistochemistry to aggrecan and type II collagen confirmed the presence of cartilage-specific ECM macromolecules in chondrogenic constructs (Fig. 2C and D, respectively). These matrix molecules were detected intracellularly and pericellularly starting as early as day 6 in chondrogenic constructs. At subsequent time points, aggrecan and type II collagen stainings were observed both intracellularly and within the lacunae enclosing chondrogenic cells. In contrast to the generalized and intense Safranin-O staining, which depicts the binding of the dye to the large number of anionic molecules in GAGs, the sparse distribution of aggrecan staining can likely be explained by relatively limited binding of the antibody to specific epitopes of the molecule.

\section{Chondrogenic conditions promote the progressive chondrocytic differentiation of hMSCs in alginate gels}

Both RT-PCR (Fig. 3A) and qRT-PCR (Fig. 3B-E) were used to assess the temporal gene expression of four key chondrocyte markers, namely, collagen types II and X, COMP, and aggrecan in the cells. These assays revealed that all four markers had temporally distinct patterns of induction in cells from chondrogenic constructs. COMP showed an early upregulation peaking at approximately 63-fold on day 12. Aggrecan mRNA levels increased significantly by day 12 , followed by a continued gradual increase until day 24 of culture. Collagen type II was highly upregulated by as much as 35-fold by day 6 and 91-fold by day 12 of culture. However, its peak induction of about 1200-fold at day 24 dwarfs this significant early upregulation. Finally, collagen type $\mathrm{X}$ showed an approximate 30 -fold induction at days 12 and 18 and peaked to a 165 -fold induction by day 24 . All four markers showed minimal changes in basal conditions, validating that the chondrogenic media indeed modulate chondrogenesis of these hMSCs.

Next, a comprehensive quantitative assessment of changes in a larger pool of chondrogenic markers in the hMSCs was performed by qRT-PCR. For the 21 genes that were examined by this method, the percent fold change in each gene was standardized relative to the peak levels considered to be $100 \%$, and the genes arranged in descending order from the one that first reaches $81-100 \%$ levels to one reaching this level last (Fig. 4). Analysis of individual genes revealed varied patterns of gene regulation over the experimental period. Thus, although many genes including COMP, HAPLN1, WNT 11, aggrecan, collagen types II and X, osteocalcin, parathyroid hormone-related protein (PTHrP), and alkaline phosphatase showed progressive increases in expression levels over the entire experimental period, other genes had bimodal patterns of induction. The genes in the latter group included collagen type VI (peaking on days 0 and 18), Sox 4 (days 0 and 18), and Sox 9 (days 12 and 24). Amongst the genes demonstrating a progressive increase over the 24 days, some such as collagen type $\mathrm{X}$ had a gradual increase in levels, while others such as matrilin 3, Ihh, Homeobox 7, chondroadherin, WNT 11, PTHrP, collagen type IX, and alkaline phosphatase demonstrated a steep upregulation. Other patterns of gene expression included a peak during mid-stages of the experiment followed by a decrease often to baseline levels, such as that for BMP-2 and collagen type I, both of which peaked at day 6. Similarly, matrilin 3, Ihh, Homeobox 7, and chondroadherin had peak expression levels at day 18 and then decreased at day 24 .

The sequential organization of the genes based on the specific time point that each first reached $81-100 \%$ expression levels enabled the derivation of a four part-staging scheme for chondrocytic differentiation of hMSCs in 3D gels (Fig. 4). This analysis revealed that collagen type VI, Sox 4, BMP-2, and collagen I were at peak levels between days 0 and 6, which was defined as stage I, while COMP, HAPLN1, collagen type XI, and Sox 9 first reached peak levels by day 12, designated as stage II. Similarly, stage III was designated when matrilin 3, Ihh, Homeobox 7, chondroadherin, and WNT 11 first attained peak levels at day 18. Finally, stage IV was designated when aggrecan; collagen types II, IX, and X; osteocalcin; fibromodulin; PTHrP; and alkaline phosphatase peaked at day 24 .

\section{Chondrogenically differentiating hMSCs in $3 D$ gels show similarities in stage-dependent profiles of chondrocyte markers to those of primary chondrocytic cells}

Using qRT-PCR, we next assessed what similarities exist between gene profiles at various stages of chondrogenic differentiation of hMSCs in 3D gels to those in primary chondrocytic cells freshly isolated from juvenile knee joint articular cartilage and adult nucleus pulposus as well as fibroblast-like cells from the annulus fibrosus (Table 2). In general, the mRNA levels for most genes assayed in the two primary chondrocytic cells were between $20 \%$ and $50 \%$ of the peak levels found in chondrogenic hMSCs. The notable exceptions to this were Sox 9, chondroadherin, and alkaline phosphatase, which had similar absolute levels in chondrogenic hMSCs and one or both types of chondrocytic cells. Additionally, matrilin 3 and osteocalcin levels were 4-fold and 20-fold greater, respectively, in nucleus pulposus cells than the peak levels in hMSCs. While the absolute levels of gene expression differed between the hMSCs and those of cells in vivo, and also in some cases between the two types of primary chondrocytic cells, an assessment of expression profiles revealed interesting relationships between the three types of primary cells and hMSCs at various stages of chondrocytic differentiation. The profiles of the select genes in day-0 hMSCs, characterized by a high expression of collagen type VI and Sox 4, and low expression of most 


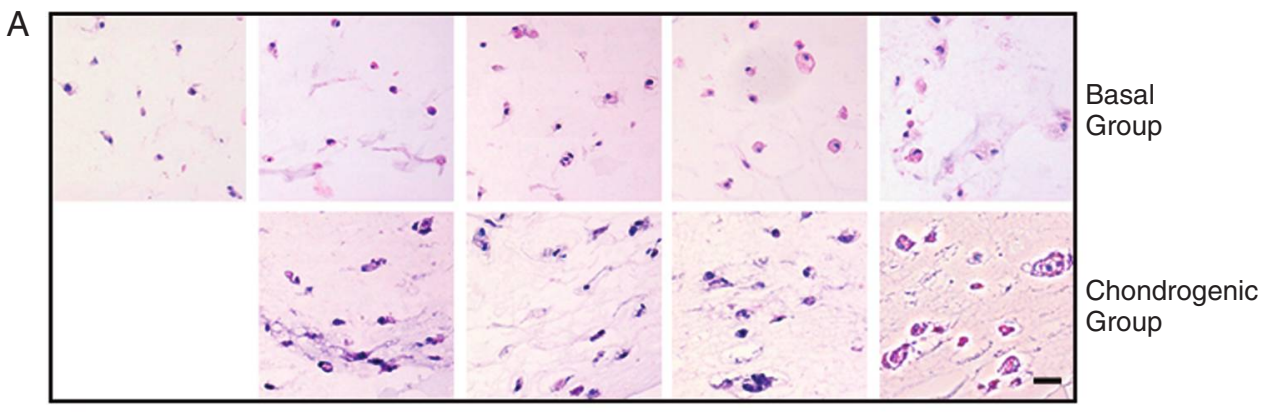

B
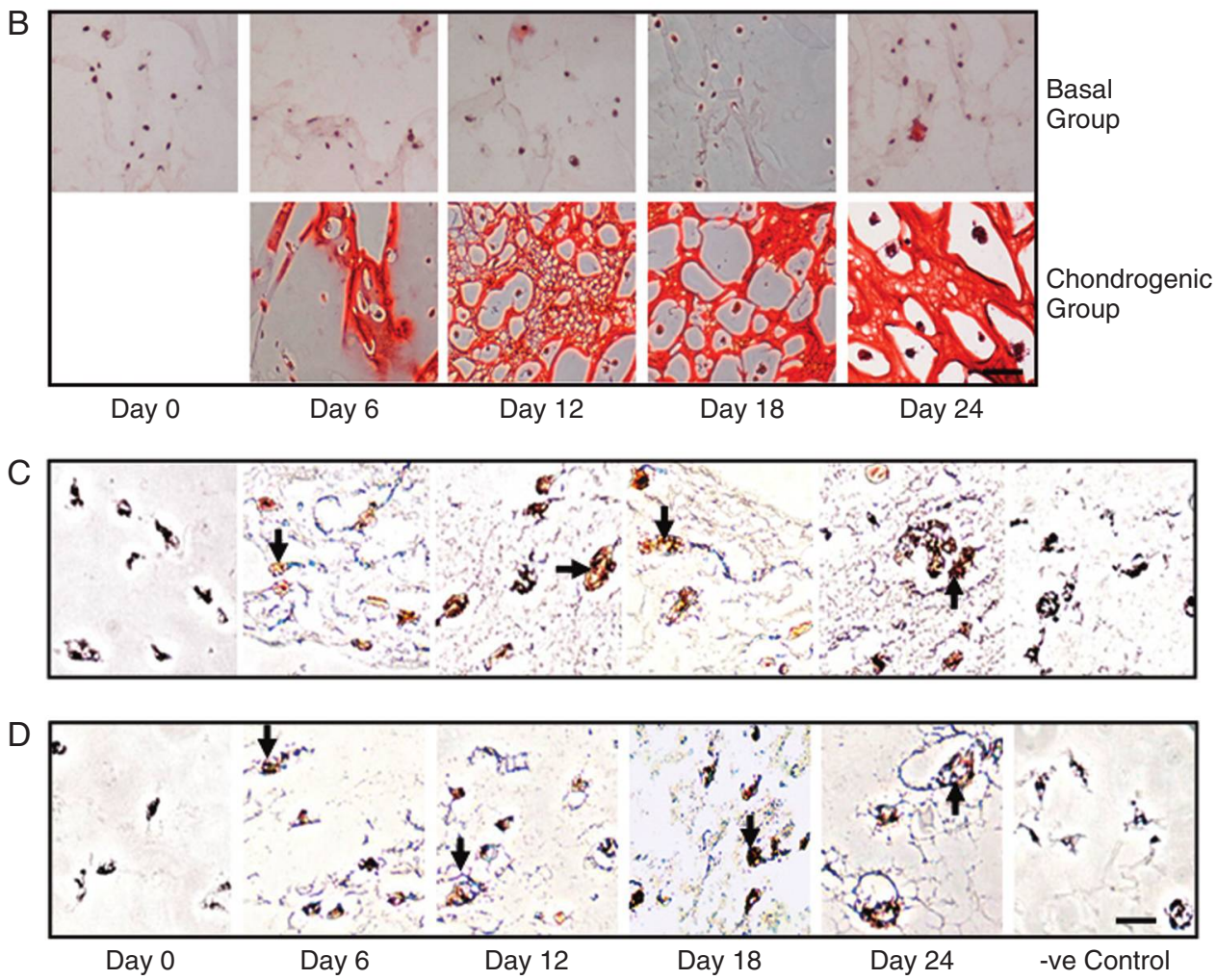

FIG. 2. hMSCs in chondrogenic constructs show distinctly different cellular morphology and distribution of cartilage-specific ECM from those in basal constructs. Cell/gel constructs were sectioned and subjected to H\&E staining and Safranin-O staining. Immunohistochemistry was also performed to detect aggrecan and collagen type II. (A) H\&E staining showed homogenous cell distribution in both basal and chondrogenic constructs, with the cells in chondrogenic media becoming progressively bigger and more rounded over 24 days of culture. Clear pericellular lacunae were also observed in the chondrogenic construct but not in the basal construct. (B) The accumulation of cartilage ECM was confirmed by Safranin-O staining, which revealed progressive increase in GAG staining in chondrogenic construct but not in basal construct during the experimental period. (C) Aggrecan and (D) collagen type II were primarily localized intracellularly at days 6 and 12 of chondrogenic cultures. At later time points, these matrix molecules showed increasing intracellular and pericellular staining to form distinguishable lacunae embracing the cells. Negative controls were incubated with nonimmune serum. (Arrows point to the positive staining; magnification $\times 20$; scale bars represent $50 \mu \mathrm{m}$.)

cartilage-related genes except WNT 11, collagen type II, and PTHrP, were most similar to those in annulus fibrosus fibroblast-like cells. Day-6 hMSCs showed gene profiles intermediate between those of annulus fibrosus cells and those from juvenile chondrocytes and nucleus pulposus cells. Exceptions to these similarities between the day- 6 hMSCs and annulus fibrosus cells were that the hMSCs showed higher levels of type XI collagen and aggrecan mRNAs than those found in the annulus fibrosus cells.
Starting from day 12 to the end of the experimental period, cells from chondrogenic hMSC constructs showed gene profiles similar to those observed in juvenile chondrocytes and nucleus pulposus cells. Exceptions to these similarities were the low levels of Homeobox 7 and alkaline phosphatase in juvenile chondrocytes, which was not the case in the day-18 and -24 chondrocytic hMSCs. Overall, the findings demonstrated that the gene profiles in hMSCs at the early stages of chondrogenic differentiation (days 0-6) resemble 
A
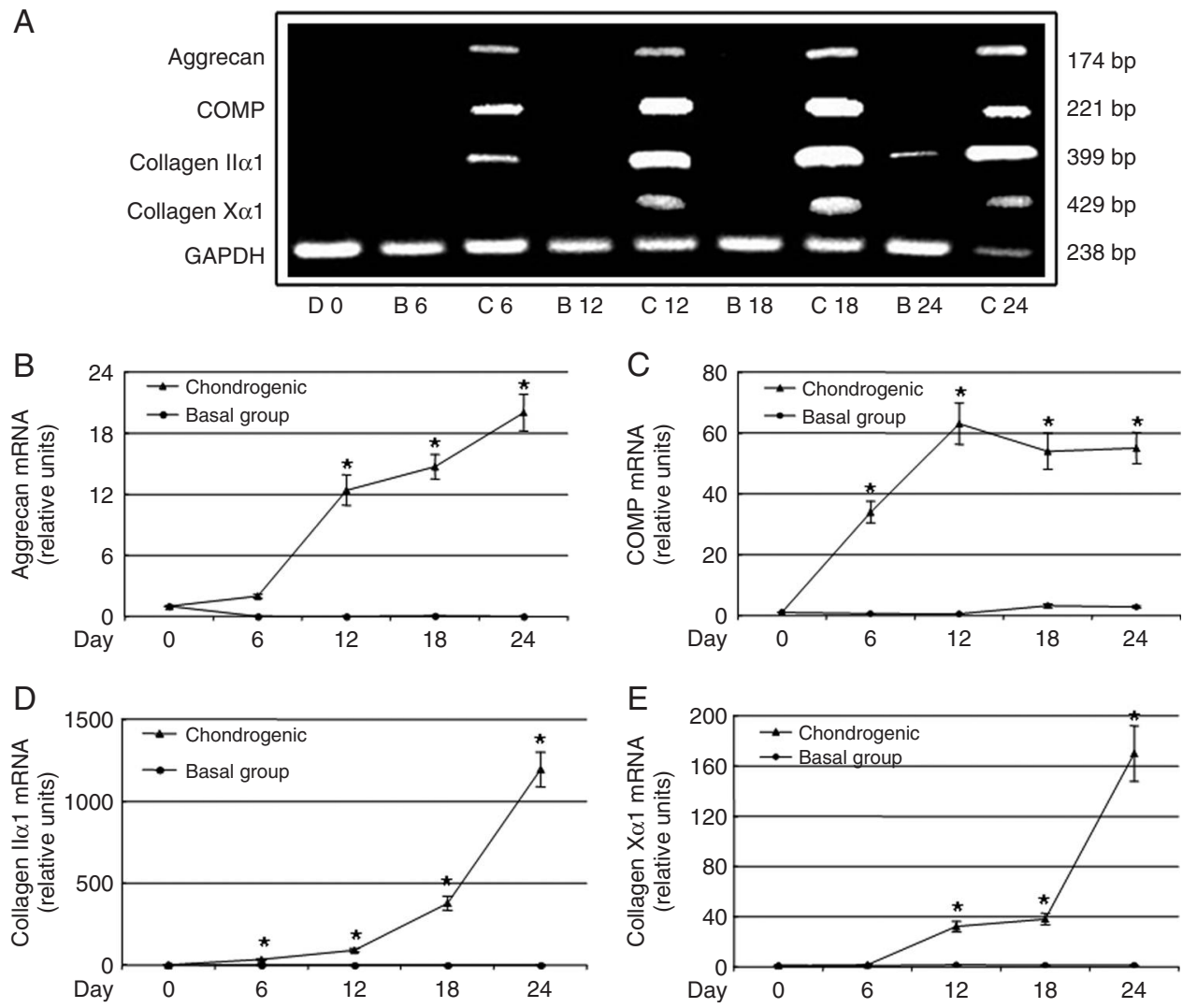

FIG. 3. Cartilage-specific genes undergo temporal upregulation during differentiation of hMSCs in 3D alginate gels. Total RNA extracted from 20 cell/gel constructs incubated in basal or chondrogenic media for up to 24 days was subjected to RT-PCR (A) and qRTPCR (B) for aggrecan, COMP, collagen types II, and X. (A) Chondrogenic constructs showed early significant increases in expression of COMP and collagen type II, which peaked at 12 and 24 days of culture, respectively. Aggrecan and type X collagen mRNA expression levels were significantly elevated by 12 days of culture and reached peak levels by 24 days. Cells incubated in basal media showed no induction in any of these markers. Data were expressed as mean $\pm \mathrm{SD}\left({ }^{*} p \leq 0.01\right.$, cells in chondrogenic vs. those in basal group at the corresponding time point).

those of fibroblast-like annulus fibrosus cells, while those of hMSCs at day 12 and after of chondrogenic differentiation had gene profiles more similar to those of chondrocytic cells.

\section{Putative novel markers for hMSC chondrogenic differentiation based on global gene expression patterns}

To identify putative novel markers unique to the individual chondrogenic stages designated in this study, we assayed global gene expression patterns at each of the four differentiation stages by microarray and developed an appropriate shortlist of genes by the analysis described in the "Materials and Methods" section. Based on historical data, of all the genes on this list, approximately one-third had some known functional link to chondrogenesis, or highly related processes, while the remainder appeared to have no prior linkage to chondrogenic processes. Since the purpose of this analysis was to identify putative novel markers as- sociated with different stages of chondrogenic differentiation, the data presented here focus on the latter set of genes. Analysis of this set of 29 stage-specific genes revealed enrichment of several functional categories relevant to chondrogenesis and/or chondrocyte physiology (Table 3). These functional categories include skeletal development (four genes), ECM (eight genes), and proteolysis/cytokine activity (four genes). A highly represented functional ontology grouping of more general interest, namely, cell communication (11 genes), was also included. Consistent with the emergence of a more highly differentiated phenotype in the latter stages of our time course, all genes linked to skeletal development, and the majority of those linked to the ECM, were upregulated at day 24. Other functional ontologies were represented by genes differentially expressed at various stages. Changes in gene expression observed in this microarray analysis trended highly toward downregulation of genes at the earliest time point (day 6) and upregulation of genes at the latest time point (day 24). 


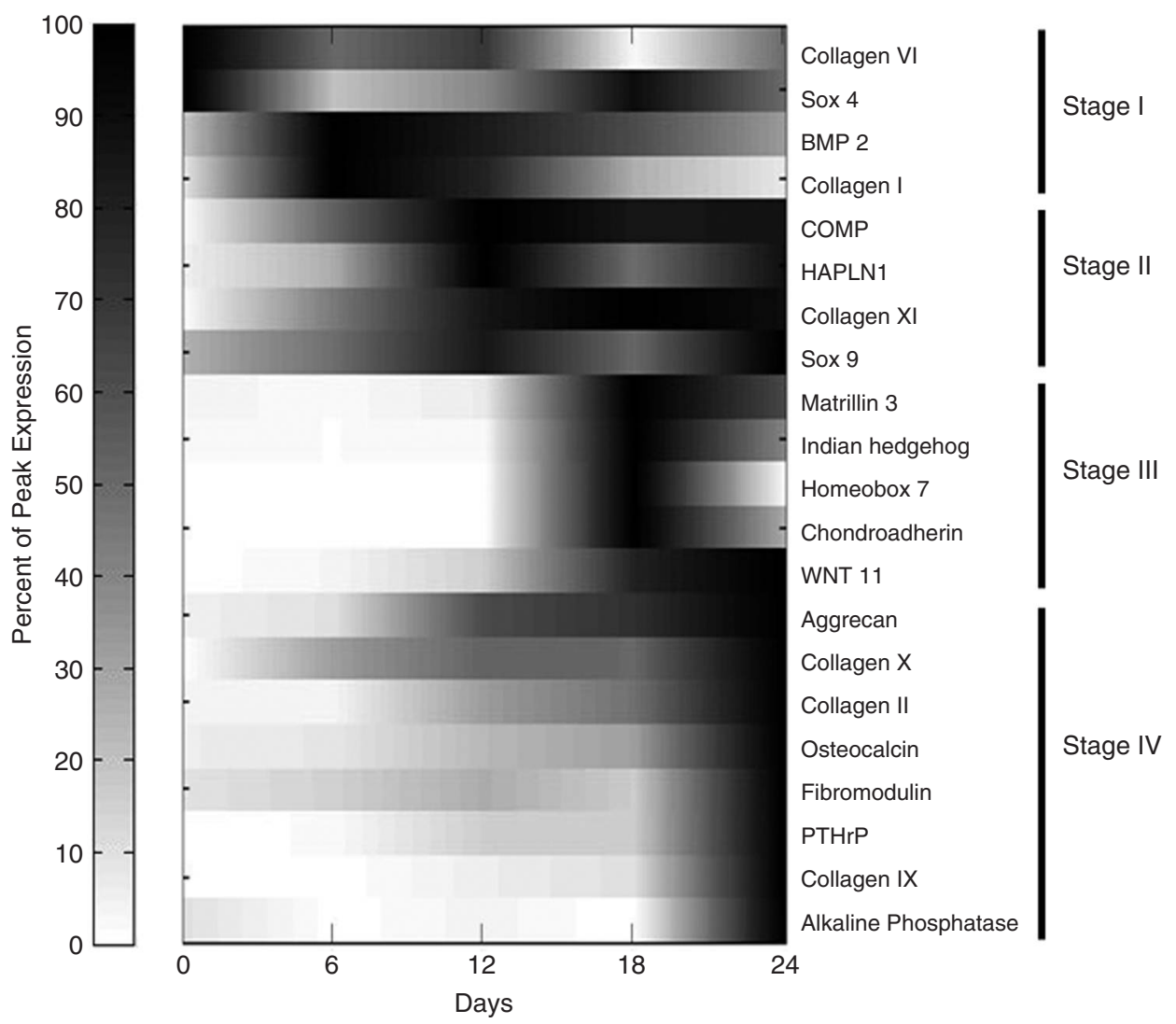

FIG. 4. Contour plot of sequential expression of chondrogenic genes in differentiating hMSCs in $3 \mathrm{D}$ gels provides a method for staging the temporal progression of chondrogenesis. RNA retrieved from chondrogenic constructs was assayed for 21 genes by qRTPCR, and the magnitude of induction of each gene was standardized to peak levels (considered 100\%). The gene expression levels were divided into $0-20 \%, 21-40 \%, 41-60 \%, 61-80 \%$, and $81-100 \%$ levels of peak levels, and plotted in gray scale with the lightest being 0-20\% and darkest being 81-100\% levels, and genes were arranged in descending order from the one that first reaches $80-100 \%$ levels to one reaching this level last. The analysis revealed both temporal differences and varied patterns of induction of individual genes over the 24-day period of the experiment. The data also enabled the differentiation to be sequenced into four stages, depicted as stages I-IV on the basis of when each gene reached its peak level.

\section{DISCUSSION}

In this study, we characterized the differentiation of hMSCs into chondrocytes under defined conditions in 3D alginate gels in vitro, and observed that temporal changes in gene expression in this system largely paralleled the spatial pattern of in vivo cartilage maturation in many important aspects. Specifically, the early gene expression profiles are similar to that in precursor cells, with the cells showing progressive increase in markers of chondroprogenitors followed by that of mature chondrocytes and then by hypertrophic chondrocytes defined by robust upregulation of collagen type $\mathrm{X}$, alkaline phosphatase, and osteocalcin. These stage-specific gene profiles show similarities at the earlier time point to those in fibroblast-like annulus fibrosus cells and in the later stages to juvenile chondrocytes and adult nucleus pulposus chondrocytic cells. The findings also provide insights into stage-specific gene profiles of hMSCs undergoing chondrogenic differentiation in vitro that may serve as a useful platform from which to explore differences in hMSC responsiveness to additional conventional and unconventional stimuli, such as mechanical load.

The ability of bone marrow stromal cells to differentiate into various cell phenotypes was first reported in studies in which colony-forming cells isolated from bone marrow were observed to differentiate under appropriate conditions into different connective tissues. ${ }^{26}$ Since then, studies have demonstrated that hMSCs are capable of differentiation along osteogenic, chondrogenic, or adipogenic lineages both in vitro and when implanted subcutaneously in SCID mice under the induction of specific bioactive agents such as growth factors, cytokines, and ECM. ${ }^{9,17,18,27}$ Among these agents, members of TGF- $\beta$ superfamily (TGF- $\beta_{1}$, TGF- $\beta_{2}$, TGF- $\beta_{3}$, and BMP-6) have proven to readily induce a sequential expression of chondrocytic markers including early induction of COMP followed by an increase in aggrecan and 


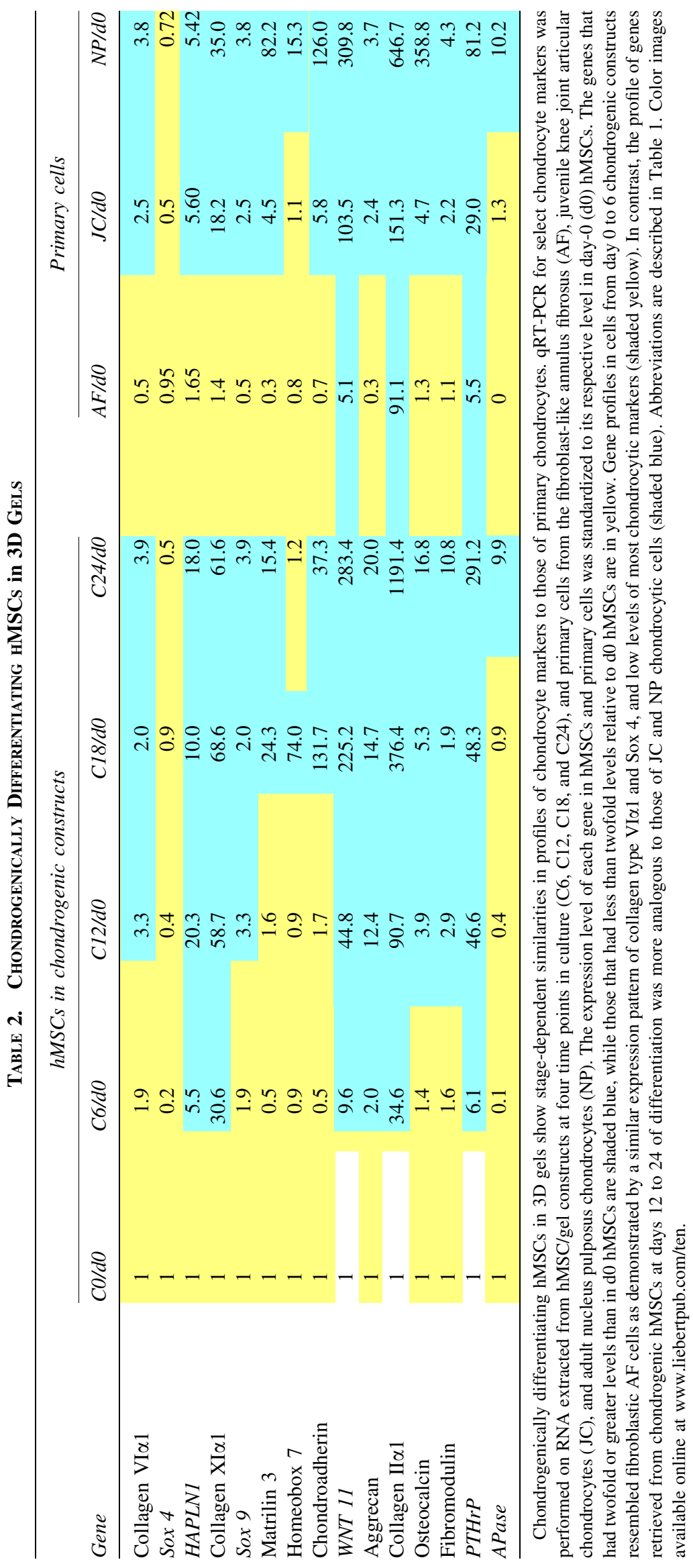


versican core protein, and subsequent upregulation of collagen types II and $\mathrm{X}$, and chondroadherin at different stages. ${ }^{28-30}$ Our studies demonstrate a similar induction of chondrogenic differentiation of hMSCs in alginate gels under defined conditions, which mimics the temporal expression pattern of cartilage-specific genes in hMSCs in pellet cultures $^{28-31}$ and in alginate gel suspension cultures. ${ }^{9,19}$

In our cell/gel constructs, the chondrogenic hMSCs assumed a rounded shape, simulating chondrocyte morphology. Additionally, the cell viability remained high throughout the experimental period, a finding consistent with previous observations. ${ }^{17,19}$ Relative to basal levels, cell proliferation was significantly diminished from days 12 to 24 in both basal and chondrogenic groups. Previous studies have shown that encapsulation of hMSCs leads to an increase in the number of cells in G0-G1 phase and a concomitant decrease in the number of cells in $\mathrm{S}$ phase.${ }^{17,19}$ This suggests that the alginate culture system can synchronize and turn off the proliferation machinery of hMSCs, perhaps by conferring upon them an appropriate 3D state. Consistent with our data, this may enable hMSCs to more readily become committed to a differentiation lineage under the defined conditions rather than continue to propagate.

Cartilage development is characterized by serial temporal and spatial changes in which chondrocytes undergo sequential morphological and metabolic transformation involving complicated regulatory and signaling networks that interweave cell-matrix and intercellular interactions with a tightly regulated gene expression pattern. ${ }^{32,33}$ This requires strict coordination between various chondrogenic factors in order to establish and maintain normal cartilage physiology. ${ }^{32,33}$ Our data suggest that sequentially regulated events that largely simulate in vivo developmental changes also occur during chondrogenic differentiation of hMSCs in alginate gels. This observation is supported not only by the differential expression over time of a variety of genes in chondrogenic hMSCs, but also by the substantial difference of cartilage-specific gene expression patterns between hMSCs cultured in chondrogenic versus basal media. Moreover, our data are in agreement with studies showing that these genes are highly expressed in chondrogenic hMSCs in both pellet culture and alginate bead systems, though no parallel time course comparison between hMSCs in chondrogenic versus basal media has been made in previous studies. ${ }^{17-19}$

Further evidence of a temporally regulated chondrogenic gene expression in hMSCs is provided by our qRT-PCR findings on 21 genes, which enabled us to characterize chondrocytic differentiation of hMSCs into four stages based on the time points when each gene first achieved 81-100\% levels. At stage I (days 0-6), collagen types I and VI, Sox 4, and BMP-2 showed peak expression levels, which were downregulated thereafter. The gene expression profile during this time period was similar to that of the primary fibroblast-like annulus fibrosus cells. During stage II, gene expression of COMP, HAPLN1, collagen type XI, and Sox 9 reached peak levels at days 6 to 12, and these were generally maintained at high expression levels at later stages. At stage III, which likely represents cells that are analogous to mature chondrocytes, gene expression of matrilin 3, Ihh, Homeobox 7 , chondroadherin, and WNT 11 peaked between days 12 and 18. Finally, between 18 and 24 days of differentiation, the cells characterized as stage-IV cells demonstrated gene profiles similar to those in hypertrophic chondrocytes with peak expression levels of aggrecan; collagen types II, IX, and X; osteocalcin; fibromodulin; PTHrP; and alkaline phosphatase. Cells at stage III and IV showed gene profiles similar to those in juvenile articular chondrocytes and adult nucleus pulposus cells.

Overall, our results are consistent with previous studies in which the temporal expression patterns of collagen types I, II, VI, IX, and X were analyzed during chondrocytic differentiation in vitro. ${ }^{34,35} \mathrm{With}$ respect to the gene expression levels from each of the two different methods (RT-PCR and microarray), trends were generally well conserved for those genes examined by both methods (data not shown). As reported in other relevant in vitro studies, upregulation of collagen type $\mathrm{X}$ in the current system appeared to be temporally advanced relative to patterns that have been historically reported in vivo. ${ }^{17,36}$ The implications of these findings from a biological as well as tissue engineering standpoint are currently unclear and only just beginning to be examined. ${ }^{37}$

As one would expect, a number of key markers, such as collagen types II and IX (COL2A1 and COL9A2), noted to be upregulated in our microarray analysis are not novel (data not shown), in that they are linked, to varying degrees, to cartilaginous tissues, or to chondrogenesis. ${ }^{38}$ Additionally, many other genes previously linked in some way to chondrocytic phenotype, including PTHLH/PTHrP, secreted frizzled-related protein 1 (SFRP1), stanniocalcin 1 (STC1), proenkephalin (PENK), CLEC3A/CLECSF1, DSPG3, frizzled-related protein, scinderin, prolactin, matrilin 4 , and gremlin, ${ }^{39-48}$ were also identified (data not shown). As a subset of these genes, those that are most commonly believed to be involved in delaying or inhibiting chondrogenesis, specifically PTHLH, SFRP1, STC1, and PENK, tended to be repressed relatively early, while those genes typically thought to be positive effectors of chondrocyte maturation, CLEC3A/CLECSF1, COL2A1, COL9A2, DSPG3, and scinderin, were induced late trends well correlated with our model of progressive cellular maturation.

Our array data also revealed the differential expression of a number of additional genes that may serve as novel stagespecific differentiation markers for chondrocytic differentiation. Thus, upregulation of scinderin, a known marker of hypertrophy detected at day 24 , together with the RT-PCR data, suggests that by day 24 , cells in our system have begun to become hypertrophic. More novelly, we posit that the observed upregulation of gremlin and downregulation of MFAP5/MAGP-2 at day 24 may be additional evidence in our array data that a hypertrophic phenotype is emerging at this stage. Expression of gremlin, a BMP antagonist, has 
been previously detected in a screen for genes expressed in cartilage growth plate chondrocytes in vivo. ${ }^{40}$ In contrast, the extracellular protein MFAP5/MAGP-2 has been recently shown to act as an agonist ligand for the notch receptor, ${ }^{49}$ and activation of this receptor by other ligands is well known to delay or block transition from the prehypertrophic to hypertrophic stage in developing chondrocytes. ${ }^{50,51}$ However, such putative roles for gremlin and MFAP5/MAGP-2 in this particular context are still entirely speculative.

\section{ACKNOWLEDGMENT}

This study was supported by NIH RO1-AR 49786 grant.

\section{REFERENCES}

1. Behonick, D.J., and Werb, Z. A bit of give and take: the relationship between the extracellular matrix and the developing chondrocyte. Mech Dev 120, 1327, 2003.

2. Buckwalter, J.A., and Mankin, H.J. Articular cartilage: degeneration and osteoarthritis, repair, regeneration, and transplantation. Instr Course Lect 47, 487, 1998.

3. Trippel, S.B. Autologous chondrocyte transplantation. N Engl J Med 332, 539, 1995.

4. Brittberg, M., Lindahl, A., Nilsson, A., Ohlsson, C., Isaksson, O., and Peterson, L. Treatment of deep cartilage defects in the knee with autologous chondrocyte transplantation. $\underline{\mathrm{N} \text { Engl J }}$ Med 331, 889, 1994.

5. Brittberg, M., Nilsson, A., Lindahl, A., Ohlsson, C., and Peterson, L. Rabbit articular cartilage defects treated with autologous cultured chondrocytes. Clin Orthop Relat Res 270, 1996.

6. Homminga, G.N., Bulstra, S.K., Bouwmeester, P.S., and van der Linden, A.J. Perichondral grafting for cartilage lesions of the knee. J Bone Joint Surg Br 72, 1003, 1990.

7. Homminga, G.N., Bulstra, S.K., Kuijer, R., and van der Linden, A.J. Repair of sheep articular cartilage defects with a rabbit costal perichondrial graft. Acta Orthop Scand 62, 415, 1991.

8. O'Driscoll, S.W., and Salter, R.B. The repair of major osteochondral defects in joint surfaces by neochondrogenesis with autogenous osteoperiosteal grafts stimulated by continuous passive motion. An experimental investigation in the rabbit. Clin Orthop Relat Res 208, 131, 1986.

9. Stevens, M.M., Qanadilo, H.F., Langer, R., and Prasad Shastri, V. A rapid-curing alginate gel system: utility in periosteum-derived cartilage tissue engineering. Biomaterials 25, 887, 2004.

10. Nehrer, S., Breinan, H.A., Ramappa, A., Shortkroff, S., Young, G., Minas, T., Sledge, C.B., Yannas, I.V., and Spector, M. Canine chondrocytes seeded in type I and type II collagen implants investigated in vitro. J Biomed Mater Res 38, 95, 1997.

11. Nehrer, S., Breinan, H.A., Ramappa, A., Young, G., Shortkroff, S., Louie, L.K., Sledge, C.B., Yannas, I.V., and Spector, M. Matrix collagen type and pore size influence behaviour of seeded canine chondrocytes. Biomaterials 18, 769, 1997.

12. Paige, K.T., Cima, L.G., Yaremchuk, M.J., Schloo, B.L., Vacanti, J.P., and Vacanti, C.A. De novo cartilage generation using calcium alginate-chondrocyte constructs. Plast Reconstr Surg 97, 168, 1996.

13. Hendrickson, D.A., Nixon, A.J., Grande, D.A., Todhunter, R.J., Minor, R.M., Erb, H., and Lust, G. Chondrocyte-fibrin matrix transplants for resurfacing extensive articular cartilage defects. J Orthop Res 12, 485, 1994.

14. Mierisch, C.M., Wilson, H.A., Turner, M.A., Milbrandt, T.A., Berthoux, L., Hammarskjold, M.L., Rekosh, D., Balian, G., and Diduch, D.R. Chondrocyte transplantation into articular cartilage defects with use of calcium alginate: the fate of the cells. J Bone Joint Surg Am 85-A, 1757, 2003.

15. Fragonas, E., Valente, M., Pozzi-Mucelli, M., Toffanin, R., Rizzo, R., Silvestri, F., and Vittur, F. Articular cartilage repair in rabbits by using suspensions of allogenic chondrocytes in alginate. Biomaterials 21, 795, 2000.

16. Sharma, B., and Elisseeff, J.H. Engineering structurally organized cartilage and bone tissues. Ann Biomed Eng 32, 148, 2004.

17. Sekiya, I., Vuoristo, J.T., Larson, B.L., and Prockop, D.J. In vitro cartilage formation by human adult stem cells from bone marrow stroma defines the sequence of cellular and molecular events during chondrogenesis. Proc Natl Acad Sci USA 99, 4397, 2002.

18. Ma, H.L., Chen, T.H., Low-Tone Ho, L., and Hung, S.C. Neocartilage from human mesenchymal stem cells in alginate: implied timing of transplantation. J Biomed Mater Res A 74, 439, 2005

19. Ma, H.L., Hung, S.C., Lin, S.Y., Chen, Y.L., and Lo, W.H. Chondrogenesis of human mesenchymal stem cells encapsulated in alginate beads. J Biomed Mater Res A 64, 273, 2003.

20. Kuo, C.K., and Ma, P.X. Ionically crosslinked alginate hydrogels as scaffolds for tissue engineering: part 1. Structure, gelation rate and mechanical properties. Biomaterials 22, 511, 2001.

21. Park, J.S., Chu, J.S., Cheng, C., Chen, F., Chen, D., and Li, S. Differential effects of equiaxial and uniaxial strain on mesenchymal stem cells. Biotechnol Bioeng 88, 359, 2004.

22. Le Maitre, C.L., Richardson, S.M., Baird, P., Freemont, A.J., and Hoyland, J.A. Expression of receptors for putative anabolic growth factors in human intervertebral disc: implications for repair and regeneration of the disc. J Pathol 207, 445, 2005.

23. Adkisson, H.D., Gillis, M.P., Davis, E.C., Maloney, W., and Hruska, K.A. In vitro generation of scaffold independent neocartilage. Clin Orthop Relat Res 391, S280, 2001.

24. Irizarry, R.A., Hobbs, B., Collin, F., Beazer-Barclay, Y.D., Antonellis, K.J., Scherf, U., and Speed, T.P. Exploration, normalization, and summaries of high density oligonucleotide array probe level data. Biostatistics 4, 249, 2003.

25. Dennis, G., Jr., Sherman, B.T., Hosack, D.A., Yang, J., Gao, W., Lane, H.C., and Lempicki, R.A. DAVID: Database for Annotation, Visualization, and Integrated Discovery. Genome Biol 4, P3, 2003.

26. Friedenstein, A.J., Petrakova, K.V., Kurolesova, A.I., and Frolova, G.P. Heterotopic of bone marrow. Analysis of precursor cells for osteogenic and hematopoietic tissues. Transplantation 6, 230, 1968.

27. Barry, F., Boynton, R.E., Liu, B., and Murphy, J.M. Chondrogenic differentiation of mesenchymal stem cells from bone marrow: differentiation-dependent gene expression of matrix components. Exp Cell Res 268, 189, 2001. 
28. Majumdar, M.K., Wang, E., and Morris, E.A. BMP-2 and BMP-9 promotes chondrogenic differentiation of human multipotential mesenchymal cells and overcomes the inhibitory effect of IL-1. J Cell Physiol 189, 275, 2001.

29. Sekiya, I., Larson, B.L., Smith, J.R., Pochampally, R., Cui, J.G., and Prockop, D.J. Expansion of human adult stem cells from bone marrow stroma: conditions that maximize the yields of early progenitors and evaluate their quality. Stem Cells 20, 530, 2002.

30. Sekiya, I., Colter, D.C., and Prockop, D.J. BMP-6 enhances chondrogenesis in a subpopulation of human marrow stromal cells. Biochem Biophys Res Commun 284, 411, 2001.

31. James, C.G., Appleton, C.T., Ulici, V., Underhill, T.M., and Beier, F. Microarray analyses of gene expression during chondrocyte differentiation identifies novel regulators of hypertrophy. Mol Biol Cell 16, 5316, 2005.

32. Kronenberg, H.M. Developmental regulation of the growth plate. Nature 423, 332, 2003.

33. DeLise, A.M., Fischer, L., and Tuan, R.S. Cellular interactions and signaling in cartilage development. Osteoarthritis Cartilage 8, 309, 2000.

34. Castagnola, P., Dozin, B., Moro, G., and Cancedda, R. Changes in the expression of collagen genes show two stages in chondrocyte differentiation in vitro. J Cell Biol 106, 461, 1988.

35. Quarto, R., Dozin, B., Bonaldo, P., Cancedda, R., and Colombatti, A. Type VI collagen expression is upregulated in the early events of chondrocyte differentiation. Development 117, 245, 1993.

36. Pelttari, K., Winter, A., Steck, E., Goetzke, K., Hennig, T., Ochs, B.G., Aigner, T., and Richter, W. Premature induction of hypertrophy during in vitro chondrogenesis of human mesenchymal stem cells correlates with calcification and vascular invasion after ectopic transplantation in SCID mice. Arthritis Rheum 54, 3254, 2006.

37. Steinert, A.F., Ghivizzani, S.C., Rethwilm, A., Tuan, R.S., Evans, C.H., and Noth, U. Major biological obstacles for persistent cell-based regeneration of articular cartilage. Arthritis Res Ther 9, 213, 2007.

38. Eyre, D. Collagen of articular cartilage. Arthritis Res 4, 30, 2002.

39. Klatt, A.R., Nitsche, D.P., Kobbe, B., Macht, M., Paulsson, M., and Wagener, R. Molecular structure, processing, and tissue distribution of matrilin-4. J Biol Chem 276, 17267, 2001.

40. Johannessen, M.K., Skretting, G., Ytrehus, B., and Roed, K.H. Neonatal growth cartilage: equine tissue specific gene expression. Biochem Biophys Res Commun 354, 975, 2007.

41. Guo, J., Chung, U.I., Yang, D., Karsenty, G., Bringhurst, F.R., and Kronenberg, H.M. PTH/PTHrP receptor delays chondrocyte hypertrophy via both Runx2-dependent and -independent pathways. Dev Biol 292, 116, 2006.
42. Gaur, T., Rich, L., Lengner, C.J., Hussain, S., Trevant, B., Ayers, D., Stein, J.L., Bodine, P.V., Komm, B.S., Stein, G.S., and Lian, J.B. Secreted frizzled related protein 1 regulates Wnt signaling for BMP2 induced chondrocyte differentiation. J Cell Physiol 208, 87, 2006.

43. Wu, S., Yoshiko, Y., and De Luca, F. Stanniocalcin 1 acts as a paracrine regulator of growth plate chondrogenesis. J Biol Chem 281, 5120, 2006.

44. Neame, P.J., Tapp, H., and Grimm, D.R. The cartilage-derived, C-type lectin (CLECSF1): structure of the gene and chromosomal location. Biochim Biophys Acta 1446, 193, 1999.

45. Deere, M., Johnson, J., Garza, S., Harrison, W.R., Yoon, S.J., Elder, F.F., Kucherlapati, R., Hook, M., and Hecht, J.T. Characterization of human DSPG3, a small dermatan sulfate proteoglycan. Genomics 38, 399, 1996.

46. Nurminsky, D., Magee, C., Faverman, L., and Nurminskaya, M. Regulation of chondrocyte differentiation by actinsevering protein adseverin. Dev Biol 302, 427, 2007.

47. Stabile, H., Mitola, S., Moroni, E., Belleri, M., Nicoli, S., Coltrini, D., Peri, F., Pessi, A., Orsatti, L., Talamo, F., Castronovo, V., Waltregny, D., Cotelli, F., Ribatti, D., and Presta, M. Bone morphogenic protein antagonist Drm/gremlin is a novel proangiogenic factor. Blood 109, 1834, 2007.

48. Ogueta, S., Munoz, J., Obregon, E., Delgado-Baeza, E., and Garcia-Ruiz, J.P. Prolactin is a component of the human synovial liquid and modulates the growth and chondrogenic differentiation of bone marrow-derived mesenchymal stem cells. Mol Cell Endocrinol 190, 51, 2002.

49. Miyamoto, A., Lau, R., Hein, P.W., Shipley, J.M., and Weinmaster, G. Microfibrillar proteins MAGP-1 and MAGP2 induce Notch1 extracellular domain dissociation and receptor activation. J Biol Chem 281, 10089, 2006.

50. Watanabe, N., Tezuka, Y., Matsuno, K., Miyatani, S., Morimura, N., Yasuda, M., Fujimaki, R., Kuroda, K., Hiraki, Y., Hozumi, N., and Tezuka, K. Suppression of differentiation and proliferation of early chondrogenic cells by Notch. J Bone Miner Metab 21, 344, 2003.

51. Crowe, R., Zikherman, J., and Niswander, L. Delta-1 negatively regulates the transition from prehypertrophic to hypertrophic chondrocytes during cartilage formation. Development 126, 987, 1999.

Address reprint requests to: Sunil Kapila, D.D.S., Ph.D. The University of Michigan 1011 North University Ave. Ann Arbor, MI 48109-1078

E-mail: skapila@umich.edu 